\title{
Pathologic characteristics of resected squamous cell carcinoma of the trachea: prognostic factors based on an analysis of 59 cases
}

\author{
Jimmie Honings $\cdot$ Henning A. Gaissert • Ruchira Ruangchira-Urai • John C. Wain • \\ Cameron D. Wright • Douglas J. Mathisen • Eugene J. Mark
}

Received: 14 August 2009 /Revised: 24 September 2009 / Accepted: 25 September 2009/Published online: 17 October 2009

(C) The Author(s) 2009. This article is published with open access at Springerlink.com

\begin{abstract}
While squamous cell carcinoma (SCC) is the most common tracheal malignancy, few reports describe the pathologic considerations that may guide intraoperative decisions and prognostic assessment. We reviewed 59 tracheal SCC treated between 1985 and 2008 by segmental resection of the trachea, including resection of the carina in $24 \%$ and inferior larynx in $14 \%$. We classified these tumors by grading histologic differentiation and microscopic features used in SCC of other sites. Of 59 tumors, 24\% (14 of 59) were well differentiated, $49 \%$ (29 of 59) were moderately differentiated, and 27\% (16 of 59) were poorly differentiated. Unfavorable prognostic factors were tumor extension into the thyroid gland (all of five so-afflicted patients died of tumor progression within 3 years) and lymphatic invasion (mean survival 4.6 versus 7.6 years). Keratinization, dyskeratosis, acantholysis, necrosis, and tumor thickness did not predict prognosis. As surgical resection is the only curative treatment; the surgeon should establish clean lines of resection using, as appropriate, intraoperative frozen section. The pathologist can provide additional important prognostic information, including tumor differentiation and extent, invasion of surgical margins, and extension into the thyroid.
\end{abstract}

J. Honings $\cdot$ H. A. Gaissert $(\bowtie) \cdot$ J. C. Wain · C. D. Wright

D. J. Mathisen

Division of Thoracic Surgery,

Massachusetts General Hospital and Harvard Medical School,

32 Fruit Street, Blake 1570,

Boston, MA 02114, USA

e-mail: hgaissert@partners.org

R. Ruangchira-Urai • E. J. Mark

Department of Pathology,

Massachusetts General Hospital and Harvard Medical School, Boston, MA, USA
Keywords Trachea $\cdot$ Squamous cell carcinoma . Airway resection · Pathologic staging .

Histologic features of invasion

\section{Introduction}

Although squamous cell carcinoma (SCC) accounts for more than half of all tracheal tumors [1], the pathologic features in resection specimens and the impact of these features on survival have only been described in small series [2]. At least two staging systems have been proposed for tracheal malignancies; one, a cross-sectional analysis of a national cancer database by Bhattacharyya, is based on retrospective tumor-node-metastases (TNM) documentation correlated with survival statistics in 41 squamous cell carcinomas, 19 adenoid cystic carcinomas, and 32 tumors of other histology [3], although a TNM staging system validated by clinical and pathologic evidence for this disease does not exist. The other, by Macchiarini, is a nonvalidated TNM classification [4]. Both classifications are intended to be applied to all histologic types of tracheal cancer.

We describe specific pathologic features of primary tracheal SCC in a large series of surgical specimens from a single institution to identify prognostic predictors of survival.

\section{Materials and methods}

A retrospective analysis was conducted of consecutive patients who underwent resection for primary SCC of the trachea from 1985 to 2008 at Massachusetts General Hospital (MGH) and for whom pathology slides or blocks were available. All except four patients were included in a previous report describing the surgical results [5]. We 


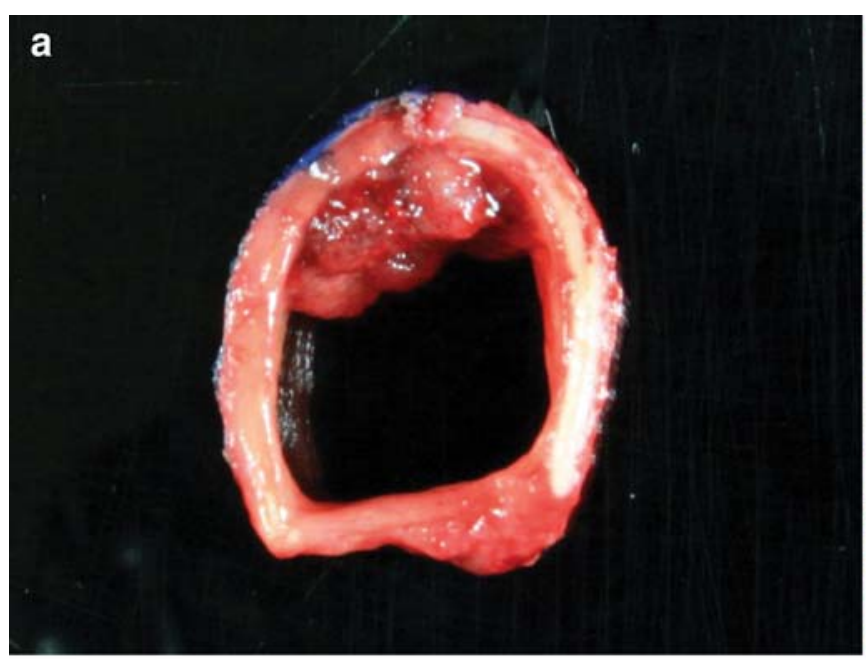

C
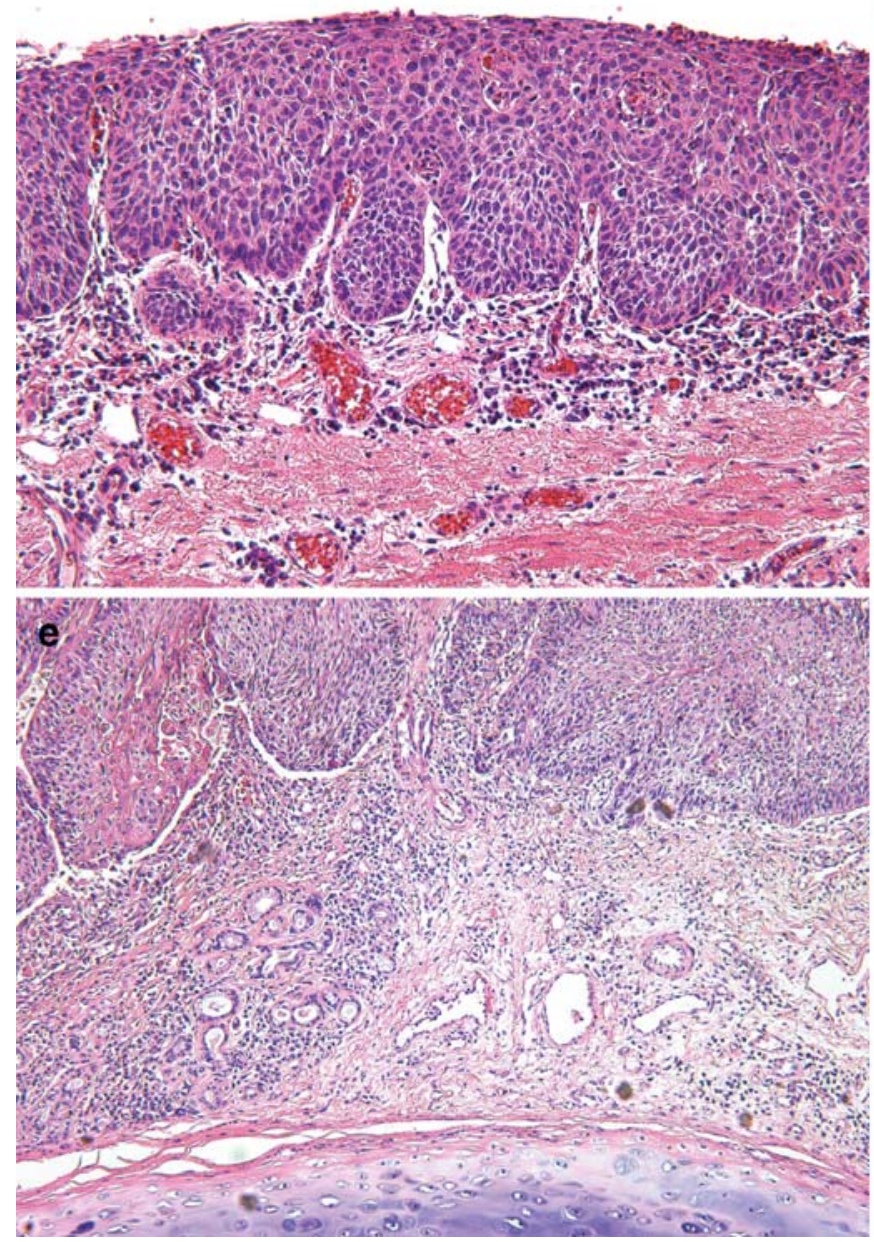

Fig. 1 a Cross section of trachea with exophitic tumor attached to anterior wall; b lymphatic invasion in peritracheal adventitia; c squamous cell carcinoma in situ, with full thickness replacement of epithelium by cells with hyperchromatic nuclei and lack of surface maturation; $\mathbf{d}$ cancerization of mucus ducts and glands by malignant
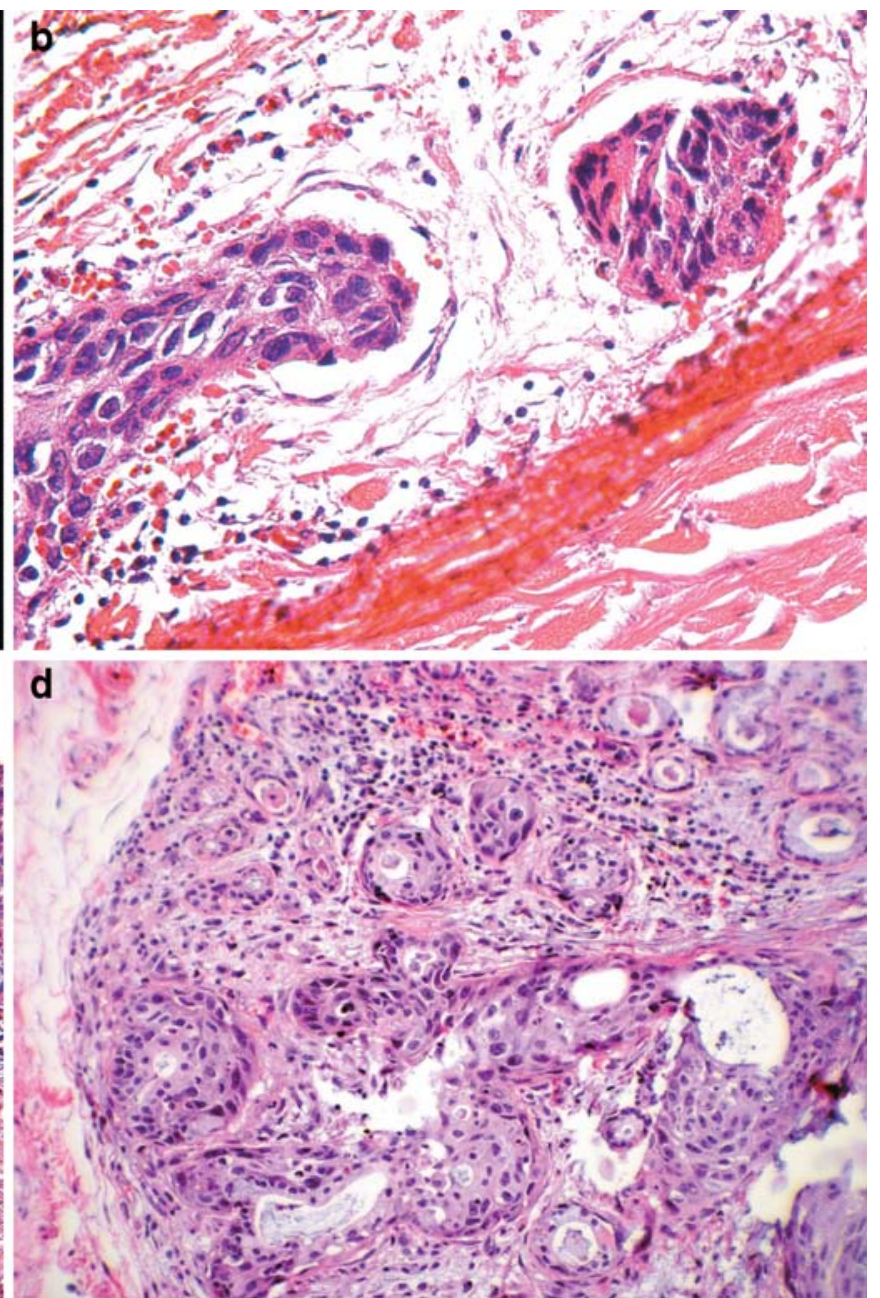

squamous cells but no invasion beyond basement membrane; e squamous cell carcinoma invading from surface epithelium into lamina propria and eliciting inflammation and fibrosis but not as far as tracheal cartilage (bottom) 


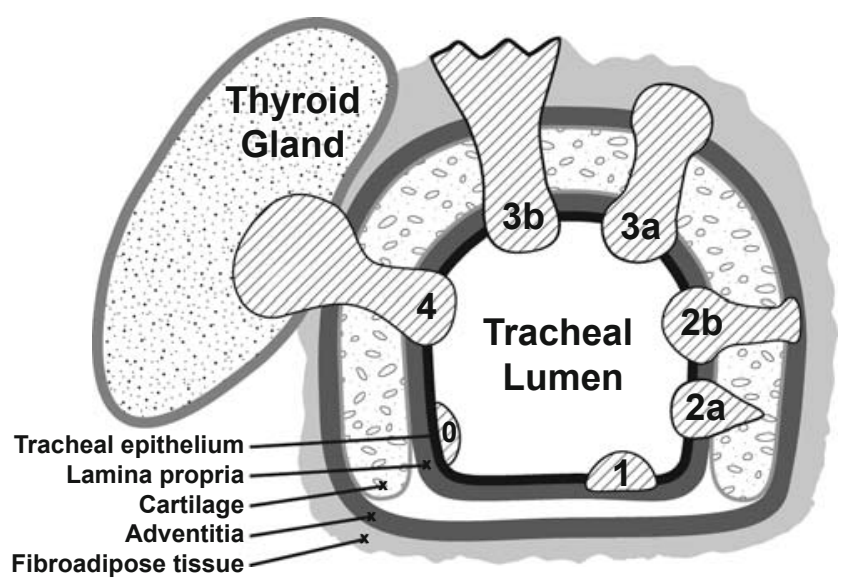

Fig. 2 Depth of invasion in tracheal squamous cell carcinoma ranked by levels. Tumor invaded between cartilage plates and not through the cartilage. Level definitions: 0 carcinoma in situ, 1 infiltrating lamina propria, $2 a$ abutting or extending between cartilage, $2 b$ invading beyond cartilage, $3 a$ invading peritracheal fibroadipose tissue, $3 b$ abutting soft tissue resection margin, 4 invading into thyroid gland

included one patient in whom a second resection at MGH completed tumor excision after a first resection elsewhere. One patient underwent three resections, but only the first, for tumor resection, was included in this study. Pathology reports, operative reports, and hospital charts were reviewed to exclude laryngeal cancer and tumors of the lung that extended to the carina. The MGH institutional review board approved the most recent protocol (no. 2008-P-000113) in January 2008.

\section{Types of resection}

The surgical technique has been detailed previously [6]. For standard tracheal resection, a sleeve of trachea was removed with end-to-end reconstruction, resulting in two airway margins and a radial soft tissue margin. When tracheal resection was combined with laryngectomy, cervical or mediastinal end-tracheostomy was required. For laryngotracheal resection, the tumor was removed with a portion of infraglottic larynx while preserving at least one recurrent laryngeal nerve not involved with tumor. Reconstruction was achieved by shaping the remaining trachea to conform to the laryngeal defect. Carinal resection was performed with or without concomitant lung resection resulting in either two or three airway margins, respectively. Systematic lymph node dissection is assumed to interfere with the tracheal blood supply and was therefore not performed. Regional lymph nodes were often not included in the specimen if not grossly enlarged. Absence of tumor at the airway margins was confirmed by frozen section, unless the limits of resection had been reached, and no additional trachea could be removed.
Pathologic review and tumor data

In all reviewed cases, photographs of the resected specimen (Fig. 1a) and slides stained with hematoxylin and eosin were retrieved. Slides were recut from stored blocks if necessary. Pathologic review of two or more slides per case was done in each case by a senior pulmonary pathologist (E.J.M.), on average four to six slides of the tumor itself and additional slides of resection margins and lymph nodes. The following histologic features were scored: degree of differentiation based on degree and extent of nuclear pleomorphism, using the criteria for well, moderately, or poorly differentiated subtypes in the Armed Forces Institute of Pathology Atlas of Tumor Pathology [7]; tumor thickness, defined as the distance from the luminal tumor surface to the farthest extent of invasion; keratinization; necrosis; dyskeratosis; acantholysis; and lymphatic invasion (Fig. 1b). Since no TNM-based staging system currently exists for tracheal carcinoma, depth of tumor invasion into the tracheal wall was scored according to a system devised for the study as shown in Fig. 2, with both squamous cell carcinoma in situ (Fig. 1c) and invasion of carcinoma into tracheal mucus glands and ducts (so-called cancerization, Fig. 1d) considered level 0 and superficially invasive (Fig. 1e) as grade 1.

Further, operative and pathology reports were reviewed for length of resection in the long axis of the airway and the presence of tumor metastasis in lymph nodes. Since lymph node excision in tracheal resection occurs merely sporadically, we combined cases with positive lymph node biopsy and cases with lymphatic invasion on histologic examination in order to provide a meaningful, albeit restricted, analysis.

\section{Adjuvant radiotherapy}

Postoperative radiotherapy was usually recommended at a dose of 54 Gy 6 to 8 weeks after resection and often administered outside Massachusetts General Hospital.

\section{Follow-up}

Patients and the MGH cancer registry were contacted for follow-up information. The Social Security Death Index was searched. Patients were determined to have died if name, date of birth, and social security number matched. The survival period began on the day of operation and was concluded by death.

Statistical analysis

Overall survival after airway resection was calculated using the Kaplan-Meier procedure with the log rank test 
Table 1 Survival according to pathologic subgroups

\begin{tabular}{|c|c|c|c|c|c|c|}
\hline \multirow[t]{2}{*}{ Pathologic subgroup } & \multirow[t]{2}{*}{ Number } & \multirow[t]{2}{*}{ Percent } & \multirow[t]{2}{*}{ Mean survival (years) } & \multirow[t]{2}{*}{$P$ value } & \multicolumn{2}{|c|}{ Survival (\%) } \\
\hline & & & & & 5 -year & 10 -year \\
\hline \multicolumn{7}{|l|}{ Tumor differentiation } \\
\hline Well differentiated & 14 & 23.7 & 8.8 & \multirow[t]{3}{*}{0.164} & 73 & 55 \\
\hline Moderately differentiated & 29 & 49.2 & 6.3 & & 44 & 25 \\
\hline Poorly differentiated & 16 & 27.1 & 4.5 & & 29 & 10 \\
\hline \multicolumn{7}{|l|}{ Keratinization } \\
\hline Yes & 39 & 66.1 & 6.5 & \multirow[t]{2}{*}{0.719} & 50 & 28 \\
\hline No & 20 & 33.9 & 6.6 & & 39 & 26 \\
\hline \multicolumn{7}{|l|}{ Necrosis } \\
\hline Extensive & 12 & 20.3 & 7.2 & \multirow[t]{3}{*}{0.726} & 46 & 27 \\
\hline Focal & 28 & 47.5 & 6.1 & & 45 & 26 \\
\hline No & 19 & 32.2 & 6.2 & & 47 & 30 \\
\hline \multicolumn{7}{|l|}{ Dyskeratosis } \\
\hline Yes & 35 & 59.3 & 6.7 & \multirow[t]{2}{*}{0.942} & 51 & 34 \\
\hline No & 24 & 40.7 & 6.3 & & 39 & 20 \\
\hline \multicolumn{7}{|l|}{ Acantholysis } \\
\hline Yes & 16 & 27.1 & 5.6 & \multirow[t]{2}{*}{0.307} & 47 & 31 \\
\hline No & 43 & 72.9 & 6.9 & & 46 & 26 \\
\hline \multicolumn{7}{|l|}{ Lymphatic invasion } \\
\hline Yes & 22 & 37.3 & 4.6 & \multirow[t]{2}{*}{0.049} & 24 & 24 \\
\hline No & 37 & 62.7 & 7.6 & & 60 & 31 \\
\hline \multicolumn{7}{|l|}{ Depth of invasion } \\
\hline Level 0 & 2 & 3.4 & n.a. & \multirow[t]{7}{*}{0.001} & 100 & 100 \\
\hline Level 1 & 5 & 8.5 & 7.6 & & 75 & 25 \\
\hline Level 2a & 14 & 23.7 & 6.0 & & 50 & 25 \\
\hline Level $2 b$ & 11 & 18.6 & 7.1 & & 50 & 38 \\
\hline Level 3a & 16 & 27.1 & 7.7 & & 53 & 31 \\
\hline Level 3b & 6 & 10.2 & 2.1 & & & \\
\hline Level 4 & 5 & 8.5 & 1.4 & & 0 & \\
\hline \multicolumn{7}{|l|}{ Tumor thickness } \\
\hline $0.1-1.0 \mathrm{~cm}$ & 29 & 49.2 & 6.8 & \multirow[t]{4}{*}{0.650} & 48 & 32 \\
\hline $1.1-2.0 \mathrm{~cm}$ & 21 & 35.6 & 6.8 & & 56 & 26 \\
\hline$>2.0 \mathrm{~cm}$ & 9 & 15.3 & 4.1 & & 13 & 13 \\
\hline Overall & 59 & 100.0 & 6.5 & & 46 & 27 \\
\hline
\end{tabular}

implemented in the SPSS 14.0 statistical software program (SPSS Inc, Chicago, IL), in contrast to the earlier report that used an actuarial method [5]. Categorical variables were compared with the chi-square test, and covariate analysis was done with Pearson's bivariate analysis.

\section{Results}

Since 1985, 75 patients have undergone surgical resection for tracheal SCC at Massachusetts General Hospital. There were no operative deaths. Slides or blocks were retrieved in 64 cases, of which five were excluded: there was no viable tumor after radiotherapy in three patients, a cutaneous SCC occurred at a tracheostomy site in one, and two separate SCC were present in one other patient, of which only one was resected. Thus, the study included 59 cases.

\section{Overall characteristics}

There were 44 men (75\%) and 15 women (25\%) with a mean age of 61.9 years (range 29 to 79 years). Prior locoregional or preoperative radiotherapy was administered in $24 \%$ (14 of 59). Resection involved trachea only in $63 \%$ (37 of 59), trachea and carina in 24\% (14 of 59), and trachea and larynx in 14\% (eight of 59) of cases. Mean length of resected airway was $3.2 \mathrm{~cm}$ (range 1 to $6 \mathrm{~cm}$ ). 

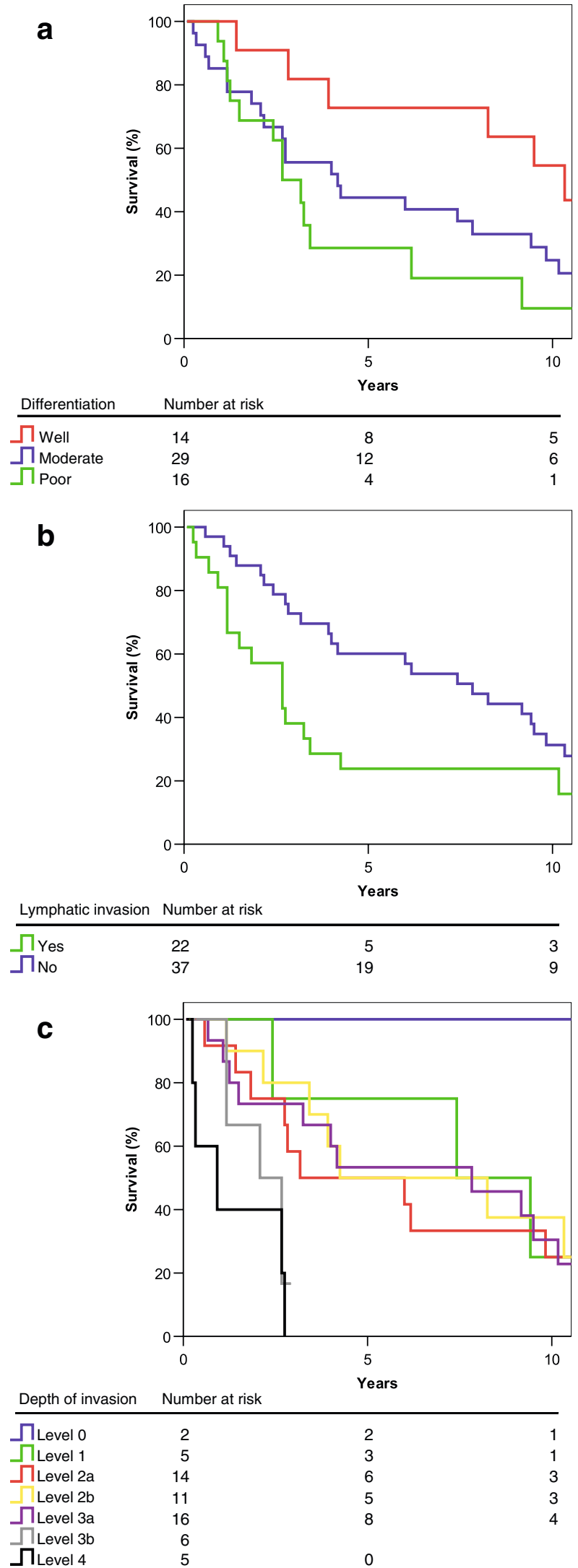

Fig. 3 a-c Overall survival according to: a tumor differentiation, b lymphatic invasion, $\mathbf{c}$ depth of tumor invasion
Pathologic characteristics are listed in Table 1. Postoperative radiation was documented in $42.4 \%$ of patients ( 25 of 59). We know of no patient who received postoperative chemotherapy.

Follow-up and overall survival

Mean follow-up in 59 cases was 5.5 years (range 1 month to 18.3 years). Survival information was complete in $90 \%$ of patients (53 of 59). Mean survival was 6.5 years, and the 5- and 10-year survivals were $46 \%$ and $27 \%$, respectively. Table 1 shows the survival calculated for each subgroup.

\section{Differentiation}

A majority of tumors had moderate differentiation, while close to half were equally distributed between well and poorly differentiated. Survival was longer in welldifferentiated tumors, though this difference was not significant (Fig. 3a). Survival in well-differentiated carcinomas was 8.8 years versus 5.8 years in the group of moderately and poorly differentiated carcinomas combined $(P=0.117)$.

Keratinization, necrosis, dyskeratosis, and acantholysis

Two thirds of tumors (39 of 59) showed keratinization. Focal or extensive necrosis was present in $48 \%$ (28 of 59 ) and 20\% (12 of 59), respectively. Necrosis was present significantly more frequently in moderately and poorly differentiated tumors $(82 \%, 37$ of 45$)$ compared to well-differentiated tumors $(21 \%$, three of 14 ; $P<0.001)$. Dyskeratosis was seen in $59 \%$ and acantholysis in $27 \%$.

Survival was not significantly correlated with the presence or absence of keratinization, necrosis, dyskeratosis, or acantholysis.

Lymphatic invasion

In 22 cases $(37 \%)$, there was tumor in peritracheal lymphatics on histologic examination (seven cases), a tumor-positive lymph node biopsy was present (nine), or both (six). In the other 36 cases (61\%), neither lymphatic invasion nor lymph node metastasis was demonstrated. Lymphatic invasion was correlated with the degree of differentiation: histologic lymphatic invasion or tumorpositive lymph node biopsies were present in one of 14 well-differentiated (7\%), 13 of 29 moderately differentiated $(49 \%)$, and nine of 16 poorly differentiated $(56 \%)$ tumors $(P=0.015)$. Survival in patients without lymphatic invasion was higher than when lymphatic invasion was present $(P=$ 
Table 2 Pathologic characteristics according to depth of invasion

\begin{tabular}{|c|c|c|c|c|c|c|c|c|}
\hline \multirow[t]{2}{*}{ Depth of invasion } & \multirow[t]{2}{*}{ Number } & \multirow[t]{2}{*}{ Percent } & \multicolumn{2}{|c|}{ Well differentiated } & \multicolumn{2}{|c|}{ Lymphatic invasion } & \multicolumn{2}{|c|}{ Tumor thickness $>2 \mathrm{~cm}$} \\
\hline & & & $N$ & $\%$ & $N$ & $\%$ & $N$ & $\%$ \\
\hline Level 0 & 2 & 3.4 & 2 & 100.0 & 0 & 0.0 & 0 & 0.0 \\
\hline Level 1 & 5 & 8.5 & 2 & 40.0 & 0 & 0.0 & 0 & 0.0 \\
\hline Level 2a & 14 & 23.7 & 5 & 35.7 & 1 & 7.1 & 0 & 0.0 \\
\hline Level 2b & 11 & 18.6 & 4 & 36.4 & 5 & 45.5 & 2 & 18.2 \\
\hline Level 3a & 16 & 27.1 & 1 & 6.3 & 7 & 43.8 & 6 & 37.5 \\
\hline Level $3 b$ & 6 & 10.2 & 0 & 0.0 & 4 & 66.7 & 1 & 16.7 \\
\hline Level 4 & 5 & 8.5 & 0 & 0.0 & 5 & 100.0 & 0 & 0.0 \\
\hline Overall & 59 & 100.0 & 14 & 23.7 & 22 & 37.3 & 9 & 15.3 \\
\hline
\end{tabular}

0.049, Fig. 3b). There was no difference in survival between patients who had lymphatic invasion only versus patients with positive lymph nodes only, versus both $(P=0.382)$.

\section{Depth of invasion}

Carcinomas in situ (level 0) were uncommon, while tumor invaded peritracheal fibroadipose tissue (level 3a) in 16 cases $(27 \%)$, extended to the soft tissue resection margin (level $3 b$ ) in six cases $(10 \%)$, and invaded the thyroid gland (level 4) in five cases ( $8 \%$; Table 2). While both patients with carcinoma in situ were alive without disease 9.6 and 15 years after resection, there was no difference in survival between levels 1, 2 ( $\mathrm{a}$ and $\mathrm{b}$ ), and 3a (Table 1 and Fig. 3c). Incomplete resection and involvement of the thyroid gland were both negative prognostic markers: mean survival was 2.1 years in patients with tumor at the soft tissue resection margin (level $3 b$ ) and 1.4 years when

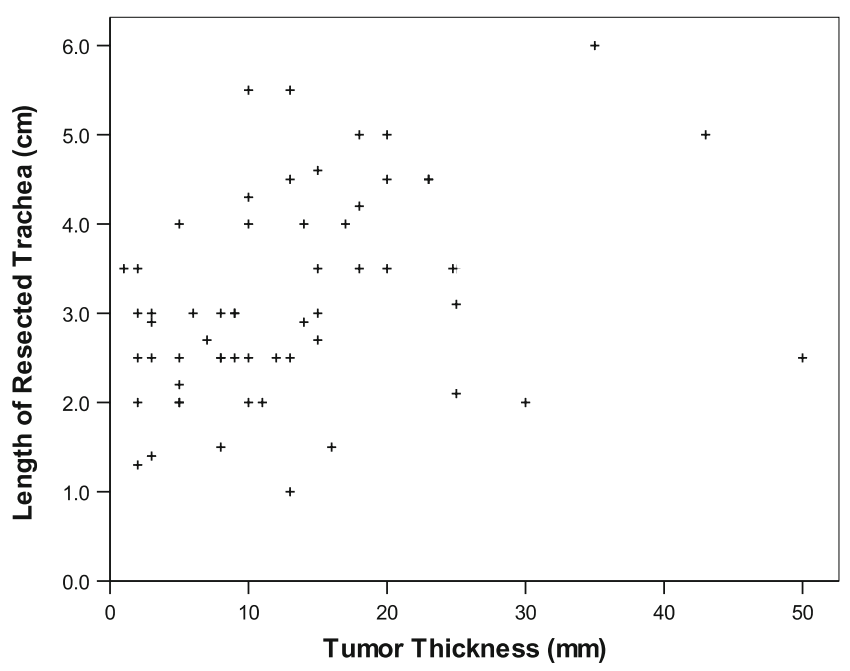

Fig. 4 Correlation between thickness and length of resected tracheal tumor. Each data point represents one tumor specimen tumor invaded the thyroid gland (level 4), both significantly lower than in other levels of invasion $(P=0.025$ and $P<0.001$, respectively). Lesser levels of invasion were associated with a higher incidence of well-differentiated histology $(P=0.018)$ and a lower incidence of lymphatic invasion $(P=0.002)$.

Tumor thickness

Mean tumor thickness was $1.3 \mathrm{~cm}$ (median $1.1 \mathrm{~cm}$, range $0.1-5.5 \mathrm{~cm}$ ). Tumors were $2 \mathrm{~cm}$ or smaller in $85 \%$. Within this range, tumor thickness had no significant impact on survival $(P=0.650)$. Tumors of greater thickness were associated with resections of greater $(>3 \mathrm{~cm})$ length $(P=$ $0.008)$, although there were two resections of 2.0 and $2.5 \mathrm{~cm}$ in length of trachea with a tumor of 3 and $5 \mathrm{~cm}$ in thickness, respectively (Fig. 4, correlation coefficient $0.364)$. Tumors $>2 \mathrm{~cm}$ in thickness had no different survival than tumors of $2 \mathrm{~cm}$ or less $(P=0.353)$. Tumors $>2 \mathrm{~cm}$ in thickness more frequently invaded peritracheal fibroadipose tissue (six of nine, 67\%) than smaller tumors (ten of 50 , $20 \% ; P=0.004)$ but were not associated with levels 3 b or 4 (Table 2).

\section{Discussion}

In this pathologic review of primary tracheal squamous cell carcinoma, the largest series to date, we show that completeness of resection, involvement of the thyroid gland, and lymphatic invasion are histopathologic features with important prognostic value. Increasing depth of invasion into the tracheal wall is associated with a loss of histologic differentiation and lymphatic invasion. Although survival in well-differentiated carcinomas is higher, this difference is not significant and may be attributed to the higher incidence of lymphatic invasion in moderately and poorly differentiated carcinomas. Further, we observed that 
resected tracheal SCCs are mostly small tumors (median thickness $1.1 \mathrm{~cm}$ ) and that tumor thickness within this range did not significantly affect prognosis. A possible explanation for this finding is that thickness may result from the exophitic portion of the tumor and thus is not conditioned on deeper invasion into the tracheal wall. A higher proportion of larger tumors, however, exhibit lymphatic invasion, a significant predictor of survival.

Surgical resection may lead to excellent survival even when the tumor violates the boundaries of the trachea and invades peritracheal fibroadipose tissue. To provide the best chances of survival, it is however important for the surgeon to achieve negative soft tissue resection margins. When faced with a tracheal SCC growing into the thyroid gland, our findings indicate that surgical resection should be applied to carefully selected patients, and palliative therapy may be considered when complete resection is otherwise compromised. Further, the pathologist reviewing SCC of the trachea should be aware of the importance of lymphatic invasion and specifically investigate the tumor for the presence of this feature.

There are some limitations to our study. We retrospectively analyzed large pathologic specimens of tracheal SCC, and only resected cases were included. Tumors growing into vital organs such as the heart or the great vessels and tumors involving long segments of airway judged unresectable were treated at the Massachusetts General Hospital but are not included in our study. We might have underestimated or misjudged the histologic characteristics, as only a limited number of representative slides for each case were available during histologic review; further, there were only five cases with invasion of the thyroid gland.

Tracheal SCC is a rare tumor, and few centers acquire proficiency in its surgical treatment. The dissemination of whatever prognostic information is available assumes therefore a greater importance. We confirm that lymphatic invasion predicts prognosis, while depth of invasion could not be correlated with survival, except for a marked decline when tumor was present at the resection margin or invaded the thyroid gland. We therefore conclude that even in cases where tumor invades peritracheal fibroadipose tissue, excellent survival can be achieved provided the patient undergoes surgical resection and the resection is complete. Positive resection margins in turn predict treatment failure. Our previous report showed that outcome in patients with unresected tumors is worse than in patients that underwent resection [5], a finding supported here by the poor prognosis in patients with incomplete resection. Thus, tumor resectability, usually dictated by tumor length in the long axis of the airway and invasion of vital organs, may possibly be the single most important prognostic factor in this very distinct type of cancer.

Conflicts of interest and funding None of the authors has had nor currently has any financial or personal relationships with other people or organizations that could inappropriately influence (bias) their work. There are no conflicts of interest. There were no sources of funding other than departmental funds.

Open Access This article is distributed under the terms of the Creative Commons Attribution Noncommercial License which permits any noncommercial use, distribution, and reproduction in any medium, provided the original author(s) and source are credited.

\section{References}

1. Gelder CM, Hetzel MR (1993) Primary tracheal tumours: a national survey. Thorax 48:688-692

2. Sweeney EC, Hughes F (1977) Primary carcinoma of the trachea. Histopathology 1:289-299

3. Bhattacharyya N (2004) Contemporary staging and prognosis for primary tracheal malignancies: a population-based analysis. Otolaryngol Head Neck Surg 131:639-642

4. Macchiarini P (2006) Primary tracheal tumours. Lancet Oncol 7:83-91

5. Gaissert HA, Grillo HC, Shadmehr MB, Wright CD, Gokhale M, Wain JC, Mathisen DJ (2004) Long-term survival after resection of primary adenoid cystic and squamous cell carcinoma of the trachea and carina. Ann Thorac Surg 78:1889-1897

6. Grillo HC (2004) Surgical techniques. In: Grillo HC (ed) Surgery of the trachea and bronchi. BC Decker, Hamilton, pp 499-692

7. Colby TV, Koss MN, Travis WD (1994) Squamous cell carcinoma and variance. In: Colby TV, Koss MN, Travis WD (eds) Atlas of tumor pathology. Tumors of the lower respiratory tract. Armed Forces Institute of Pathology, Washington, DC, pp 157-167 